ARTICLE

https://doi.org/10.1038/s41467-019-13519-1

\title{
Amorphization activated ruthenium-tellurium nanorods for efficient water splitting
}

Juan Wang ${ }^{1,4}$, Lili Han²,4, Bolong Huang (10 ${ }^{3 \star}$, Qi Shao ${ }^{1}$, Huolin L. Xin (iD ${ }^{2}$ \& Xiaoqing Huang ${ }^{1 \star}$

Pursuing active and durable water splitting electrocatalysts is of vital significance for solving the sluggish kinetics of the oxygen evolution reaction (OER) process in energy supply. Herein, theoretical calculations identify that the local distortion-strain effect in amorphous $\mathrm{RuTe}_{2}$ system abnormally sensitizes the Te-p $\pi$ coupling capability and enhances the electrontransfer of Ru-sites, in which the excellent inter-orbital p-d transfers determine strong electronic activities for boosting OER performance. Thus, a robust electrocatalyst based on amorphous $\mathrm{RuTe}_{2}$ porous nanorods (PNRs) is successfully fabricated. In the acidic water splitting, a-RuTe 2 PNRs exhibit a superior performance, which only require a cell voltage of $1.52 \mathrm{~V}$ to reach a current density of $10 \mathrm{~mA} \mathrm{~cm}{ }^{-2}$. Detailed investigations show that the high density of defects combine with oxygen atoms to form $\mathrm{RuO}_{x} \mathrm{H}_{y}$ species, which are conducive to the OER. This work offers valuable insights for constructing robust electrocatalysts based on theoretical calculations guided by rational design and amorphous materials.

\footnotetext{
${ }^{1}$ College of Chemistry, Chemical Engineering and Materials Science, Soochow University, 215123 Jiangsu, China. ${ }^{2}$ Department of Physics and Astronomy, University of California, Irvine, CA 92697, USA. ${ }^{3}$ Department of Applied Biology and Chemical Technology, The Hong Kong Polytechnic University, Hung Hom, Kowloon, Hong Kong SAR. ${ }^{4}$ These authors contributed equally: Juan Wang, Lili Han. *email: bhuang@polyu.edu.hk; hxq006@suda.edu.cn
} 
E lectrochemical water splitting, emerging as an attractive technology for generating hydrogen $\left(\mathrm{H}_{2}\right)$ and oxygen $\left(\mathrm{O}_{2}\right)$, provides a potential to address environmental degradation and energy crises due to its pure and clean products ${ }^{1-4}$. Noblemetal $\mathrm{Pt}$ and $\mathrm{Ir} / \mathrm{Ru}$ are considered to be the benchmark catalysts for the hydrogen evolution reaction (HER) and the oxygen evolution reaction (OER), respectively ${ }^{5-7}$. However, a high voltage is still needed to drive the reaction process because of the sluggish kinetics for OER, especially in acidic conditions ${ }^{8-11}$. Significant efforts have been undertaken to design novel catalysts to overcome this obstacle, including heteroatoms doping, functionalization, and so on ${ }^{12-16}$. Although much progress in developing bifunctional electrocatalysts for alkaline water splitting has been realized, the overpotential and corrosion resistance are still far from satisfactory under harsh acidic conditions, which hamper the development of proton exchange membrane water electrolyzers ${ }^{17-19}$.

Recent studies have been primarily focused on enhancing the intrinsic activity to improve catalytic efficiency, such as phase and interface engineering, creating grain boundaries and regulating electronic structures ${ }^{20-24}$. It should be noteworthy that those resulting catalysts are overwhelmingly based on crystalline materials and tend to ignore their amorphous counterparts. Amorphous materials are generally entities, in which the arrangement of their internal atoms is not periodic but only bear the local short-range order. The inherent disorderliness of amorphous materials can produce abundant "dangling bonds" and defects in the loosely bonded atomistic free-volume zones, which can provide more active sites and thus improve catalytic activity ${ }^{25-27}$. In addition, the unique structure and isotropic properties endow amorphous materials strongly corrosion resistance in both acidic and alkaline conditions, providing fresh insights into the search for highly stable catalysts ${ }^{28,29}$. For example, Zhang et al. prepared amorphous lithium-incorporated palladium phosphosulfide nanodots (Li-PPS NDs) by electrochemically lithiated layered $\mathrm{Pd}_{3} \mathrm{P}_{2} \mathrm{~S}_{8}$ crystal. Interestingly, this amorphization process can activate the electrochemically inert $\mathrm{Pd}_{3} \mathrm{P}_{2} \mathrm{~S}_{8}$, thereby significantly enhances its HER activity ${ }^{30}$. The obtained amorphous Li-PPS NDs also possess excellent stability under the acidic condition that the decay of current density is negligible after 10000 potential cycles. Inspired by the above, catalyst designs based on amorphous materials are therefore an attractive strategy for developing highly active and stable electrocatalysts for water splitting under harsh environments.

Herein, guided by the theoretical mechanism study of the intrinsic high electroactivity revealed in the amorphous structure, the a-RuTe $e_{2}$ porous nanorods (a-RuTe $e_{2}$ PNRs) with bullet-like outline have been designed and synthesized as robust water splitting electrocatalysts. Density functional theory (DFT) calculations reveal that high amorphization degree renders the local short-range disorder to be distinguished, which inevitably induces distortion-strain effect (DS) and thus leads the system to be the meta-stable state. Such energetic trend not only facilitates the variation of local Te-coordination for flexible bonding but also induces a medium-to-long range $p \pi$ coupling to efficiently annihilate the notorious crystal-field-splitting effect of $\mathrm{Ru}$ for highly active intra- and inter-orbital electron-transfer. From the view of strong electron-lattice coupling effect, the short-range disorder contributes to the intrinsic guarantee for high OER activities within $\mathrm{pH}$-universal conditions. As an electrocatalytic result, the a-RuTe $e_{2}$ PNRs present a significantly improved OER performance than its crystalline counterparts in harsh environments, especially in $0.5 \mathrm{M} \mathrm{H}_{2} \mathrm{SO}_{4}$. In detail, it is capable of delivering an overpotential of as low as $245 \mathrm{mV}$ for OER, far better than those of crystalline $\mathrm{RuTe}_{2}$ PNRs (c-RuTe 2 PNRs) and the benchmark electrocatalyst Ir/C. By constructing a-RuTe $\mathrm{P}_{2}$ PNRs as a two-electrode system in the acidic electrolyte, only a cell potential of $1.52 \mathrm{~V}$ is needed to generate $10 \mathrm{~mA} \mathrm{~cm}^{-2}$. Experimental results demonstrate that the distorted $\mathrm{Ru}$-Te bond can be derived from the high density of defects. Additionally, the $\mathrm{RuO}_{\mathrm{x}} \mathrm{H}_{\mathrm{y}}$ species form by combining the defect with oxygen atoms also contribute to the reaction process.

\section{Results}

DFT theoretical simulations. Although strain effect has been utilized to enhance the electron transfer activity to facilitate the electrocatalysis, the corresponding characterization still remains a huge challenge in the amorphous materials. In order to investigate the intrinsic activity of amorphous samples, we have carried out DFT calculations to evaluate the OER process of amorphous $\mathrm{RuTe}_{2}$ system before experiments. From the model, the weighted average coordination $(\mathrm{CN})$ of $\mathrm{Ru}$ is lowered $(\mathrm{CN}=6$ for crystalline $\mathrm{RuTe}_{2}$ ) staying between 4 (tetrahedral) and 6 (octahedral), while Te sites are more flexible ranged from $\mathrm{CN}=2$ to $\mathrm{CN}=6$ with Te-Te bonds (Fig. 1a). The bonding and anti-bonding orbitals near the Fermi level $\left(\mathrm{E}_{\mathrm{F}}\right)$ demonstrate a $\mathrm{p}-\pi$ electron-rich character given by Te-sites, indicating the high electronic sensitivities of Te to couple O-2p orbital for $\mathrm{H}_{2} \mathrm{O}$ activation (Fig. 1b). The line-up of reciprocal, highly symmetrical points in both crystalline and amorphous structures indicates the different electron transfer paths within the Brillouin zone, supporting the distinct electron transfer ability (Fig. $1 c, d$ ). As short-range $\mathrm{Ru}-\mathrm{Te}$ disordered, the intrinsic DS strengthens the driving force on the electronic activities. The Lamé parameters reflect the correlation within homogenous, isotropic, and continuum medium. With increasing DS-effect, the instabilities of Ru-Te bonding environment are enlarged. However, as the energetic trend reflects, Te-Te homopolar bond formation plays a key role in facilitating widerrange relaxations towards higher stabilities, supporting the dominant role of the highly sensitive $p-\pi$ coupling in both energetic performance and electronic activities (Fig. 1e). Moreover, the dielectric function of the amorphous structure is obviously larger than the crystalline structure, especially the static dielectric function $\varepsilon_{1}$. The imaginary part $\varepsilon_{2}$ of the dielectric function of the amorphous reaches the first peak value at $0.440 \mathrm{eV}$, which is much smaller than $2.60 \mathrm{eV}$ of the crystalline, confirming the much smaller electron transition from the top of valence band to the bottom of conduction band near the reciprocal, highly symmetrical points (Fig. 1f).

To further distinguish the intrinsic difference in electronic contribution of the crystalline and amorphous structures in boosting the catalysis, the band structures are plotted. The detailed theoretical derivation and discussion of the intrinsic electronic contribution enhancement of the amorphous structure in catalysis is provided in the Supplementary Note 1. Notably, the crystalline structure shows an evident indirect band gap of $0.620 \mathrm{eV}$, which demonstrates the energy barriers for $d$ - $d$ and $d-p$ transitions (Fig. 1g). In comparison, the amorphous $\mathrm{RuTe}_{2}$ exhibits electron-rich feature crossing the Fermi level without any energy gap, supporting the facile $d$ - $d$ electron transition for achieving superior OER performance. Moreover, the forbidden $p$ - $d$ electron transition in the crystalline structure has been loosen by the amorphous structure due to the induced intrinsic DS effect (Fig. 1h, i). Therefore, these results indicate that such different electron transfer leads to the contrast selectivity of OER process. Meanwhile, the projected partial density of states (PDOSs) reveal that $\mathrm{Ru}-4 \mathrm{~d}-\mathrm{t}_{2 \mathrm{~g}}$ activity (valence-peak) and valence-band-centers $\left(E_{\mathrm{V}}-1.2 \mathrm{eV}\right.$ and $E_{\mathrm{V}}-1.0 \mathrm{eV}$ for amorphous and crystal system respectively, $E_{\mathrm{V}}=0$ for $\mathrm{E}_{\mathrm{F}}$ ) remain while the DS-effect relaxes the forbidden rule of intra-orbital $e_{g}-t_{2 g}$ electron-transfer, which annihilates the gap between the $e_{g}-t_{2 g}$ splitting of the $\mathrm{Ru}-4 \mathrm{~d}$ band 
a

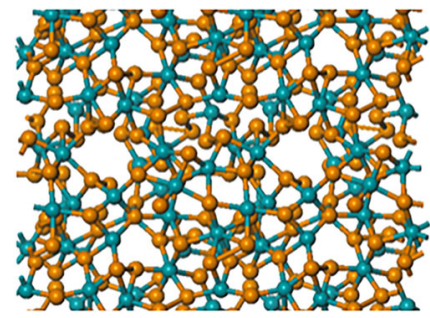

b

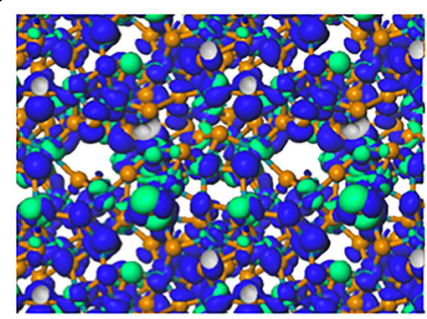

C

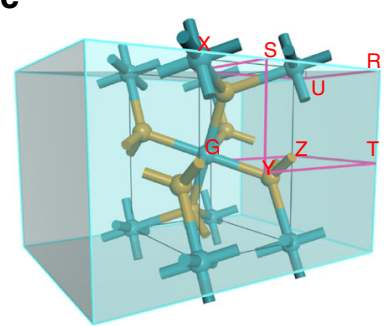

d

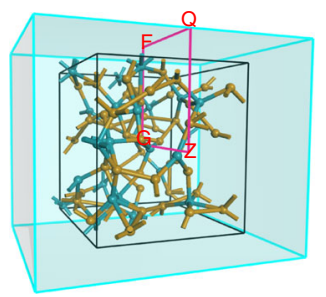

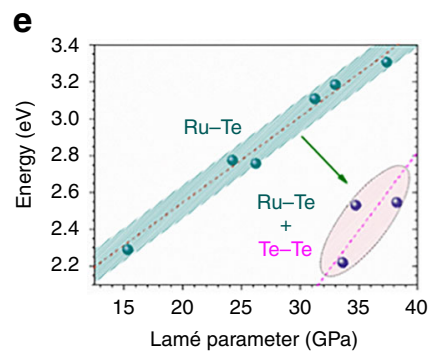
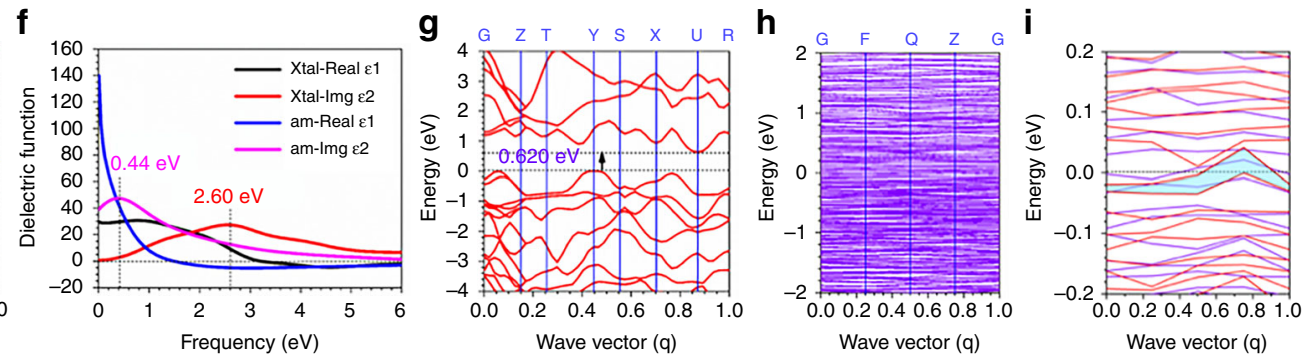

Fig. 1 Electronic structures comparison between the crystalline and amorphous RuTe $\mathbf{2}_{2}$ a Local atomic configurations for the amorphous RuTe $e_{2} \mathbf{b}$ The real spatial contour plots for bonding and anti-bonding orbitals near $E_{F}$. c The Brillouin zone of the crystalline RuTe 2 , in which the $G, Z, T, Y, S, X, U$, and $R$ denote high-symmetry points within the reciprocal space (red). d The Brillouin zone of the amorphous RuTe 2 , in which the G, F, Q, and Z denote highsymmetry points within the reciprocal space (red). e Structural formation energy variation trend with related to the Lamé parameters for describing the bulk lattice distortion induced strain effect, as well as the Te-Te bond formation. $\mathbf{f}$ The dielectric function comparison. $\mathbf{g}$ The band structure of the crystalline RuTe $e_{2}$ and $\mathbf{h}$ the amorphous RuTe$e_{2}$. i Enlarged display of the amorphous RuTe $\mathrm{H}_{2}$ band structure.
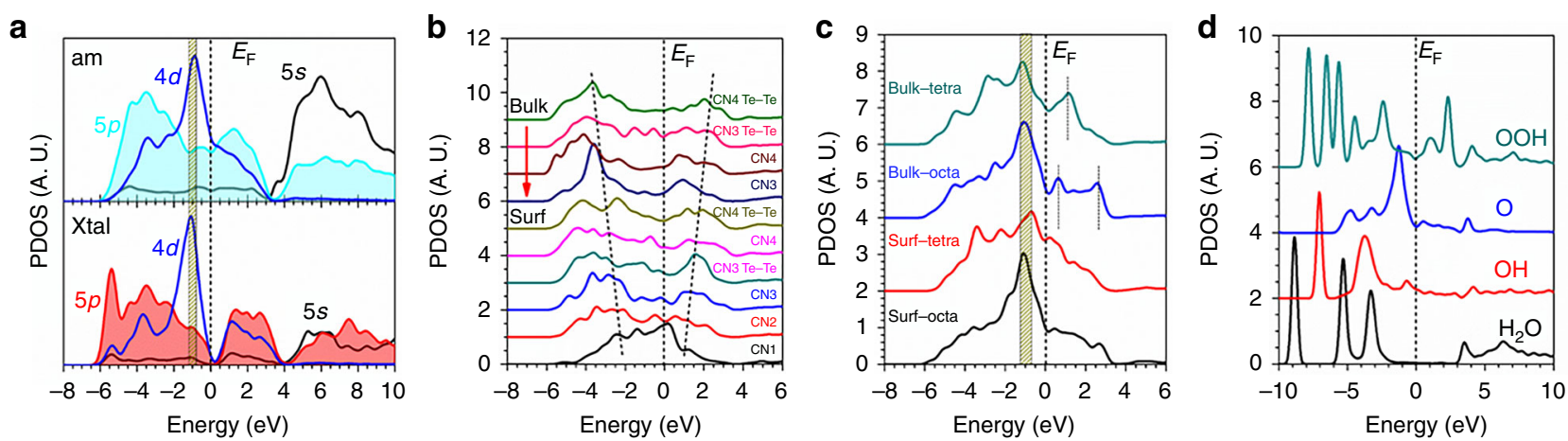

e
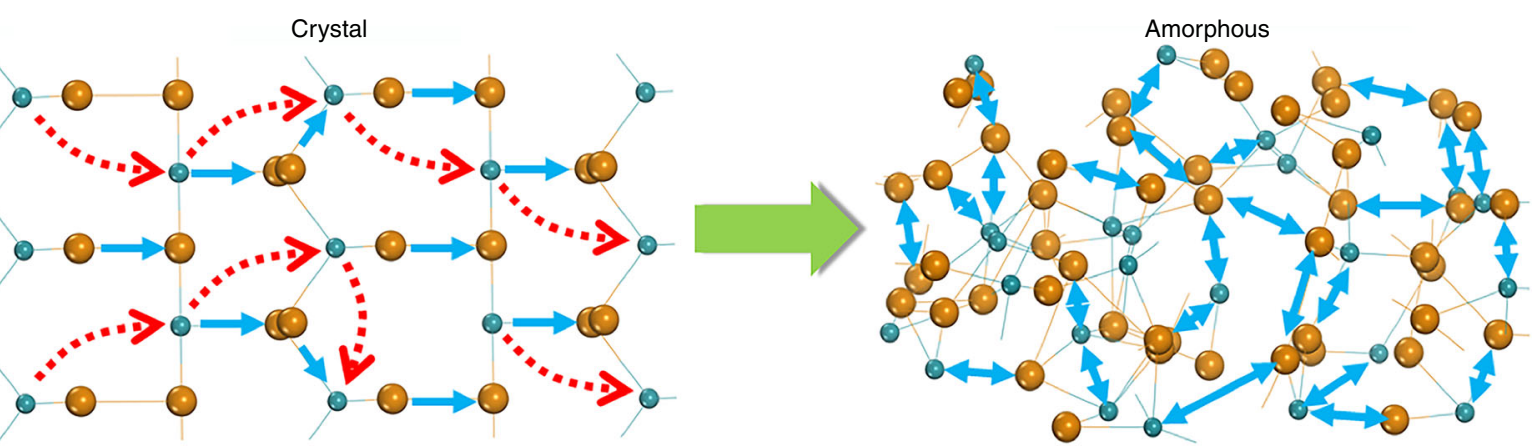

Limited d-d transfer

Flexible electron transfer

Fig. 2 Electronic activities of the amorphous $\mathbf{R u T e}_{\mathbf{2}}$ for OER. a PDOSs comparison between amorphous and crystalline RuTe $\mathrm{e}_{2}$ systems. $\mathbf{b}$ Site-dependent PDOSs variation trend of Te-5p band. c Site-dependent PDOSs variation of Ru- $4 \mathrm{~d}$ band. $\mathbf{d}$ Individual PDOSs contributions of O-species from OER process. e Schematic diagram of the electronic activity enhancement in amorphous structure.

in the amorphous structure. The distorted $\mathrm{Ru}$-Te lattice increases the homogeneity for efficient inter-d-orbital electron-transfer ability among $\mathrm{Ru}$ sites (Fig. 2a). The surface Te-5p dominantly occupies from $E_{\mathrm{V}}-4.5 \mathrm{eV}$ to $E_{\mathrm{F}}$ with the most overlapping with $\mathrm{Ru}-4 \mathrm{~d}$, enhancing the activities of $\mathrm{p}$ - $\mathrm{d}$ coupled electron-exchange.
The stronger electron transfer between $p$ - $d$ than the $p-p$ also supports the anti-oxidation of the $\mathrm{RuTe}_{2}$. The enhanced bonding and anti-bonding splitting effect of Te-5p band in the bulk region with higher $\mathrm{CN}$ results in gradually inert and less overlapped with Ru-4d (Fig. 2b). The surface $\mathrm{Ru}-4 \mathrm{~d}$ band obviously merges the 

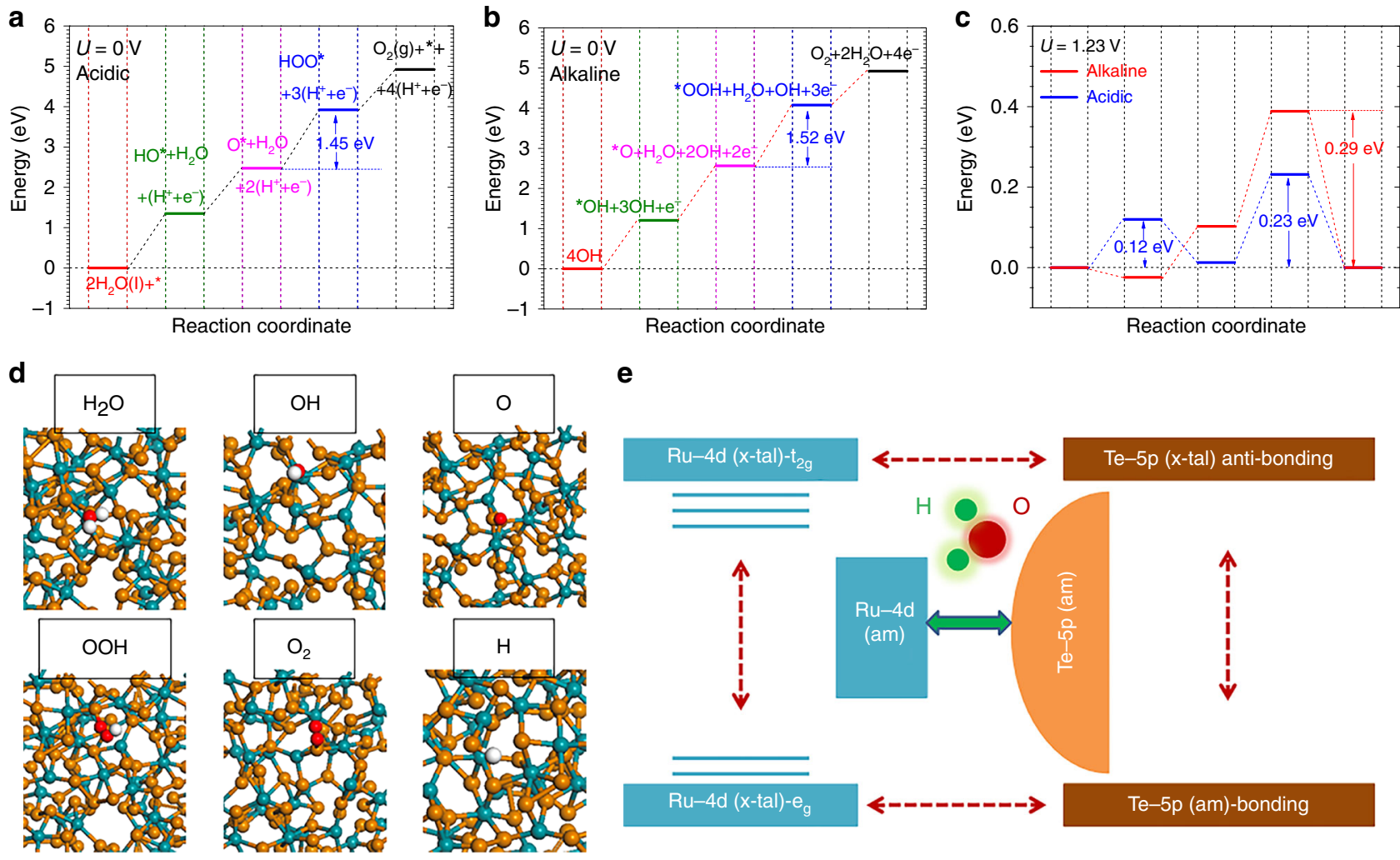

e

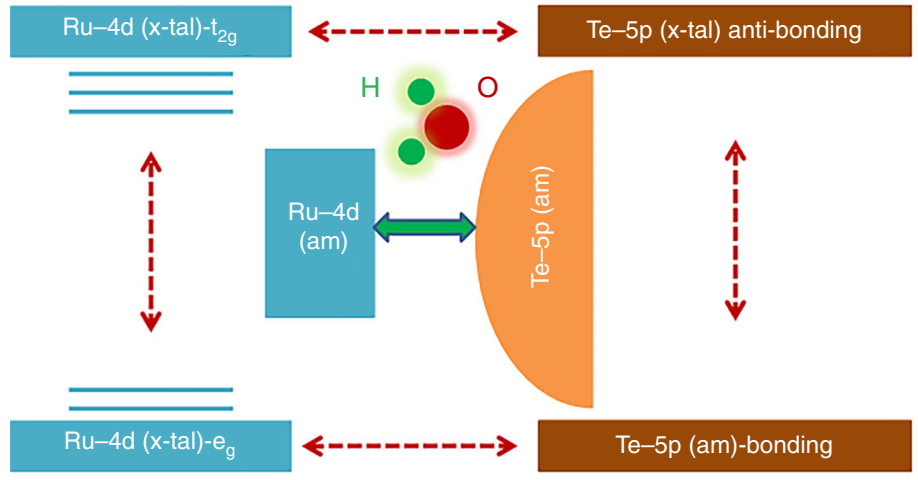

Fig. 3 OER energy profile and activity plot. a The free energetic pathways for acidic OER at $U=0 \mathrm{~V}$. The corresponding reaction mechanism in the acidic is shown in the plot and calculation setup. $\mathbf{b}$ The alkaline OER pathway at $U=O \mathrm{~V}$. The different reaction mechanisms with the acidic OER based on key adsorbates and co-reactants is supplied in the plot and calculation setup. c The OER pathways of acidic and alkaline conditions at $U=1.23 \mathrm{~V}$. $\mathbf{d}$ Local structural configurations of initial reactant, intermediates or final product on the amorphous RuTe $e_{2}$ surface. e Schematic diagram for illustrating the amorphous $\mathrm{RuTe}_{2}$ boosting the OER performance via highly efficient electron-transfer site-independently.

electronic occupations, resulting in the annihilation of the $\mathrm{e}_{\mathrm{g}}-\mathrm{t}_{2 \mathrm{~g}}$ splitting character on both surfaces octahedral and tetrahedral bonding sites. Therefore, the surface distorted $\mathrm{Ru}$-Te bonding minimizes the crystal-field splitting effect for the surface $\mathrm{Ru}$-sites (Fig. 2c). The $\mathrm{p}-\pi$ orbital of $\mathrm{H}_{2} \mathrm{O}$ exhibits a substantial overlapping with Te-5p bands showing a strong $p-\pi$ coupling to activate the $\mathrm{H}_{2} \mathrm{O}$ molecule for efficient splitting. From $\mathrm{OH}$ to $\mathrm{O}$, the $p-\pi$ coupling becomes stronger with the increase of p-orbital electronic activities. Further on the $\mathrm{OOH}$, a slightly weaker electronic activity is demonstrated due to the hydrogenation effect for O-p $\pi$ passivation (Fig. 2d). The schematic diagram of the electron transfer pathways has been presented to show the enhanced electronic activity of the amorphous structure of $\mathrm{RuTe}_{2}$ in promoting the catalysis (Fig. 2e).

We move onto the OER pathways (Fig. 3a-c). In acidic condition, the $\mathrm{Ru}-\mathrm{Te}$ surface performs an excellent $\mathrm{H}_{2} \mathrm{O}$ splitting with the substantially low activation energy for $\left[{ }^{*} \mathrm{OH}+\mathrm{H}^{+}+\mathrm{e}^{-}\right]$. The secondary de-hydrogenation performs at even lower energetic level. This trend guarantees the potential determining step to form $\mathrm{OOH}^{-}$favoring at a lower energy of $3.92 \mathrm{eV}$ relative to initial $\mathrm{H}_{2} \mathrm{O}$ level (Fig. 3a). In alkaline condition, the favorable bonding of both $\mathrm{OH}$ and $\mathrm{O}$ facilitates the $\mathrm{OOH}$ formation with a lower barrier of $1.52 \mathrm{eV}$ (Fig. 3b). Under the $U=1.23 \mathrm{~V}$ potential, the overpotentials (i.e. $\max \{[$ barrier $-1.23 \mathrm{eV}] / \mathrm{e}\}$ ) are $0.23 \mathrm{~V}$ (acidic) and $0.29 \mathrm{~V}$ (alkaline), respectively (Fig. 3c). From local structure perspectives, the $\mathrm{H}_{2} \mathrm{O}$ stabilizes between the $\mathrm{Ru}$ and Te sites, while $\mathrm{OH}, \mathrm{O}$, and $\mathrm{OOH}$ uniquely adsorbs on distorted $\mathrm{Te}$ sites, where the local Te-CN flexibly varied (Fig. 3d). We confirm that OER performance originates from surface DS-effect, which sensitizes Te-p $\pi$ coupling and annihilates the crystal-field splitting effect of Ru for highly active intra- and inter-orbital electron-transfer (Fig. 3e).

Catalyst synthesis and characterization. To realize the highly electroactive amorphous surface for OER as the theoretical predictions, we present the fabrication strategy with the rational design by following the guidance of the DFT calculations. We have successfully designed, prepared and compared $\mathrm{RuTe}_{2}$ PNRs in both amorphous and crystalline conditions. The pristine amorphous $\mathrm{RuTe}_{2}$ PNRs were prepared by a simple hydrothermal process, in which potassium tellurite $\left(\mathrm{K}_{2} \mathrm{TeO}_{3}\right)$ and hexaammineruthenium (III) chloride $\left(\mathrm{Cl}_{3} \mathrm{H}_{18} \mathrm{~N}_{6} \mathrm{Ru}\right)$ were used as metal precursors ${ }^{31}$. High-angle annular dark-field scanning transmission electron microscopy (HAADF-STEM) image shows that the products exhibit one-dimensional (1D) nanorods structure with high dispersion (Fig. 4a). TEM images show that the nanorods have a porous structure and bullet-like profile with the average diameter and length of $17.0 \pm 1.8 \mathrm{~nm}$ and $99.7 \pm 5.8 \mathrm{~nm}$, respectively (Supplementary Fig. 1). The $\mathrm{Ru} / \mathrm{Te}$ molar ratio was determined to be around 1:2 by inductively coupled plasma atomic emission spectrometry (ICP-AES) and scanning electron microscopy energy-dispersive X-ray spectroscopy (SEM-EDS). No significant diffraction peaks were observed by powder X-ray diffraction (PXRD) (Fig. 4b), demonstrating that the RuTe 2 PNRs are amorphous, corresponding to the high-resolution TEM (HRTEM) and selected area electron diffraction (SAED) (Supplementary Fig. 2). STEM-EDS element mappings reveal that the $\mathrm{Ru}$ and Te are evenly distributed along the RuTe $\mathrm{P}_{2}$ PNRs (Fig. 4c). Subsequently, the $\mathrm{RuTe}_{2}$ PNRs were loaded on VC-X72 carbon 


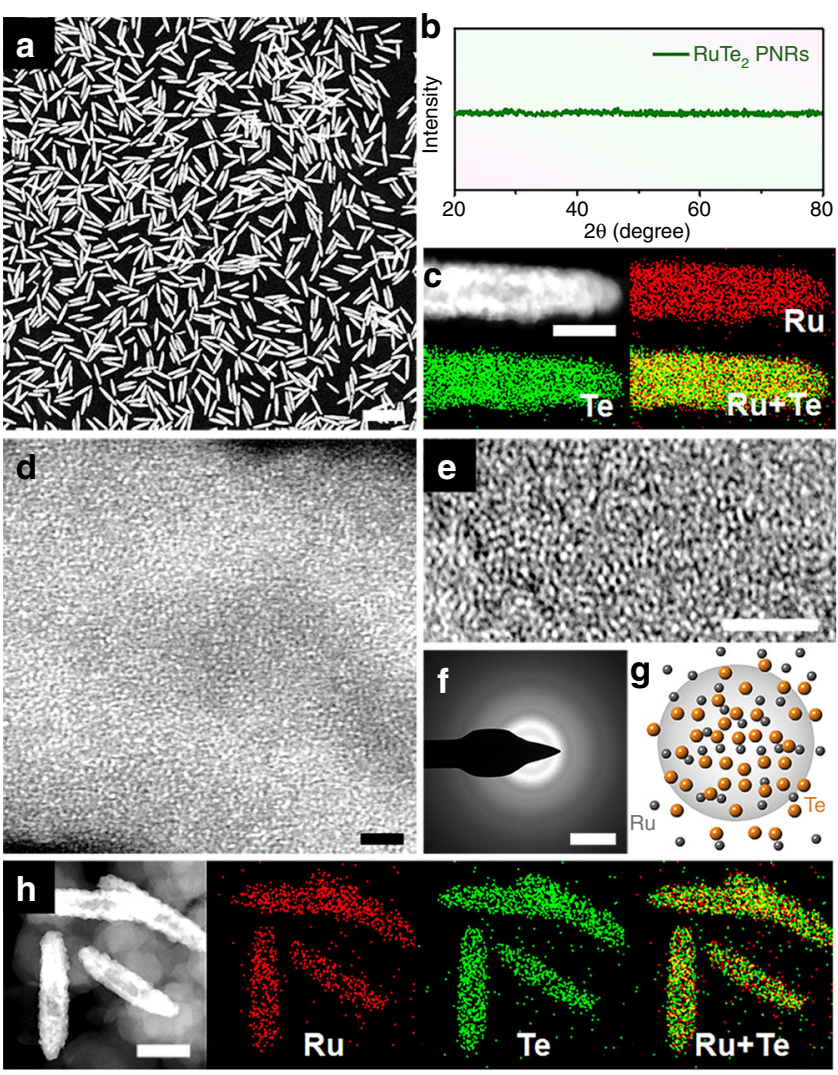

Fig. 4 Morphology and composition profile analysis. a HAADF-STEM image, $\mathbf{b}$ XRD pattern, c STEM-ADF image and EDS elemental mappings of $\mathrm{RuTe}_{2}$ PNRs. d Atomic-resolution HAADF-STEM image, e HRTEM image, f SAED pattern, $\mathbf{g}$ structural model, and $\mathbf{h}$ STEM-ADF image and EDS elemental mappings of carbon supported a-RuTe $\mathrm{e}_{2}$ PNRs. Scale bar, a $200 \mathrm{~nm}$; c $10 \mathrm{~nm}$; d, e $2 \mathrm{~nm}$; f $51 / \mathrm{nm}$, and h $40 \mathrm{~nm}$.

(Supplementary Fig. 3) and then thermally treated under air atmosphere 32,33 , where only a broad carbon peak can be found in their XRD pattern, demonstrating that the $\mathrm{RuTe}_{2}$ PNRs still maintain an amorphous structure (denoted as a-RuTe $e_{2}$ PNRs) (Supplementary Fig. 4). HAADF-STEM and TEM images show that the PNRs keep 1D porous nanorod profile with a slight increase in diameter and length. As revealed by the atomicresolution HAADF-STEM image, the atoms exhibit a random distribution without significant periodicity, proving the structural disorder of a-RuTe $e_{2}$ PNRs (Fig. 4d). Moreover, only blurred areas with no clear long-range ordered atomic arrangements can be observed from HRTEM, further demonstrating the amorphous feature of a-RuTe $e_{2}$ PNRs (Fig. 4e). SAED pattern also reveals a set of distinct rings composed of diffraction spots (Fig. 4f), being in agreement with the XRD results. The structural model of $\mathrm{RuTe}_{2}$ PNRs with the amorphous feature was successfully described (Fig. 4g), where the $\mathrm{Ru}$ and $\mathrm{Te}$ atoms exhibit a random arrangement with isotropic, short-range order in atomistic freevolume zones. STEM-EDS element mappings reveal that the $\mathrm{Ru}$ and Te are still distributed uniformly throughout the whole PNRs (Fig. 4h).

The highly crystalline $\mathrm{RuTe}_{2}$ PNRs (denoted as c-RuTe $\mathrm{P}_{2} \mathrm{PNRs}$ ) were obtained by thermal treating $\mathrm{RuTe}_{2}$ PNRs under controlled thermal treatments ${ }^{34}$. PXRD pattern shows two main peaks at $31.29^{\circ}$ and $43.37^{\circ}$, corresponding to the (111) and (211) planes of $\mathrm{RuTe}_{2}$ (PDF\#88-1380), respectively (Fig. 5a). The crystal characteristics and long-range order of $\mathrm{c}-\mathrm{RuTe}_{2}$ PNRs can also be demonstrated by atomic-resolution HRTEM image (Fig. 5b). Significantly, a large number of regularly arranged atoms can be
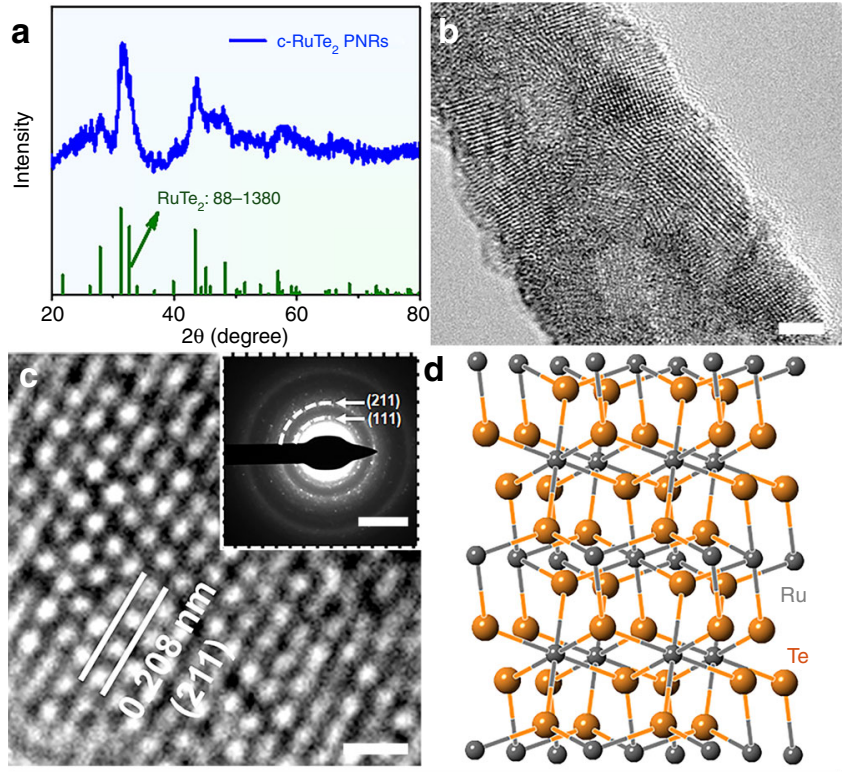

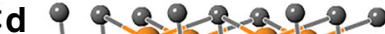
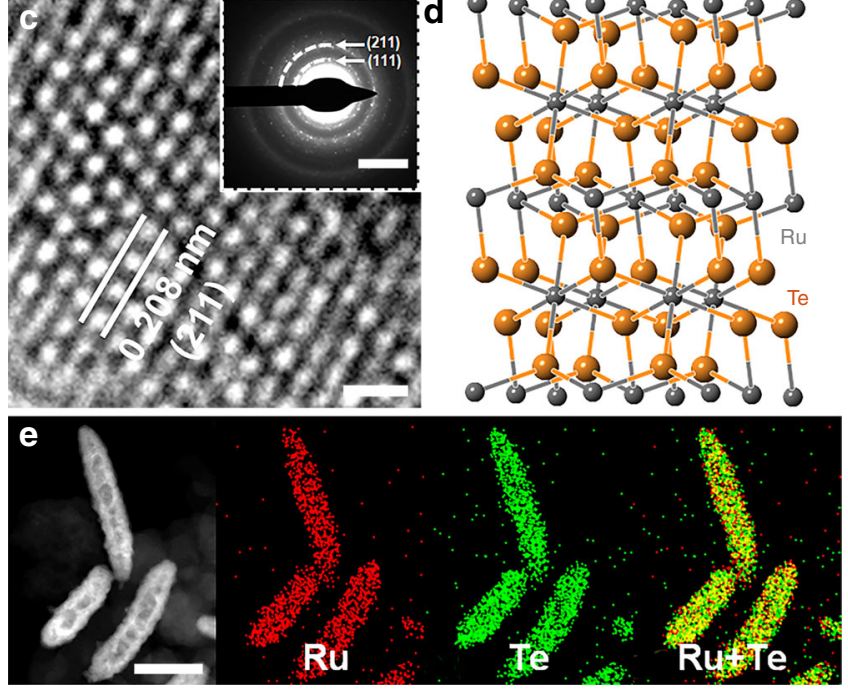

Fig. 5 Morphology and structural characterizations. a PXRD pattern, b, c HRTEM image. d structural model and e STEM-ADF image and EDS elemental mappings of carbon supported c-RuTe $e_{2}$ PNRs. Inset in $\mathbf{c}$ is the SAED pattern of c-RuTe 2 PNRs. Scale bar, b $2 \mathrm{~nm}$; c $0.5 \mathrm{~nm}$; (Inset in c) $5 \mathrm{l} / \mathrm{nm}$; e $40 \mathrm{~nm}$.

observed by HRTEM and their interplanar spacing is $0.208 \mathrm{~nm}$, corresponding to the (211) plane of $\mathrm{RuTe}_{2}$ (Fig. 5c). SAED pattern also reveals a set of distinct rings composed of diffraction spots (inset in Fig. 5c), being assigned to the (111) and (211) planes. Surprisingly, the morphology and composition of c-RuTe PNRs are largely maintained, indicating that the controlled thermal treatments only affect the crystallinity but hardly cause morphology and composition changes (Supplementary Fig. 5). The structural model of $\mathrm{c}-\mathrm{RuTe}_{2}$ PNRs presents a long-range ordered anisotropy with a regular orthorhombic structure (Pnnm) (Fig. 5d and Supplementary Fig. 6). STEM-EDS elements mappings show that the $\mathrm{Ru}$ and $\mathrm{Te}$ are uniformly distributed within the whole PNRs (Fig. 5e).

Evaluation of electrochemical activity. We performed OER and HER measurements of a-RuTe $e_{2}$ PNRs and c-RuTe $e_{2}$ PNRs under the universal $\mathrm{pH}$ range. Commercial $\mathrm{Ir} / \mathrm{C}$ and $\mathrm{Pt} / \mathrm{C}$ were tested under the same conditions for comparison. As expected, the a$\mathrm{RuTe}_{2}$ PNRs show enhanced electrocatalytic performance than that of their crystalline counterparts. In detail, as shown in Fig. 6a, the a-RuTe $e_{2}$ PNRs exhibit the best OER activity with a low overpotential of $245 \mathrm{mV}$ at $10 \mathrm{~mA} \mathrm{~cm}^{-2}$ in $0.5 \mathrm{M} \mathrm{H}_{2} \mathrm{SO}_{4}$, which is superior to those of c-RuTe $\mathrm{PNRs}_{2}(442 \mathrm{mV})$ and $\mathrm{Ir} / \mathrm{C}$ $(323 \mathrm{mV})$. Obviously, compared with $\mathrm{Ir} / \mathrm{C}(297 \mathrm{mV})$ in $1.0 \mathrm{M}$ $\mathrm{KOH}$, only an overpotential of $285 \mathrm{mV}$ is required for a- $\mathrm{RuTe}_{2}$ PNRs, while the $\mathrm{c}-\mathrm{RuTe}_{2}$ PNRs need a higher overpotential of $458 \mathrm{mV}$ under the same conditions, which is highly consistent with the theoretical predictions (Fig. $6 \mathrm{~b}$ and Supplementary Table 1). Importantly, the a-RuTe 2 PNRs not only present excellent electrocatalytic performance in the strong acidic and 

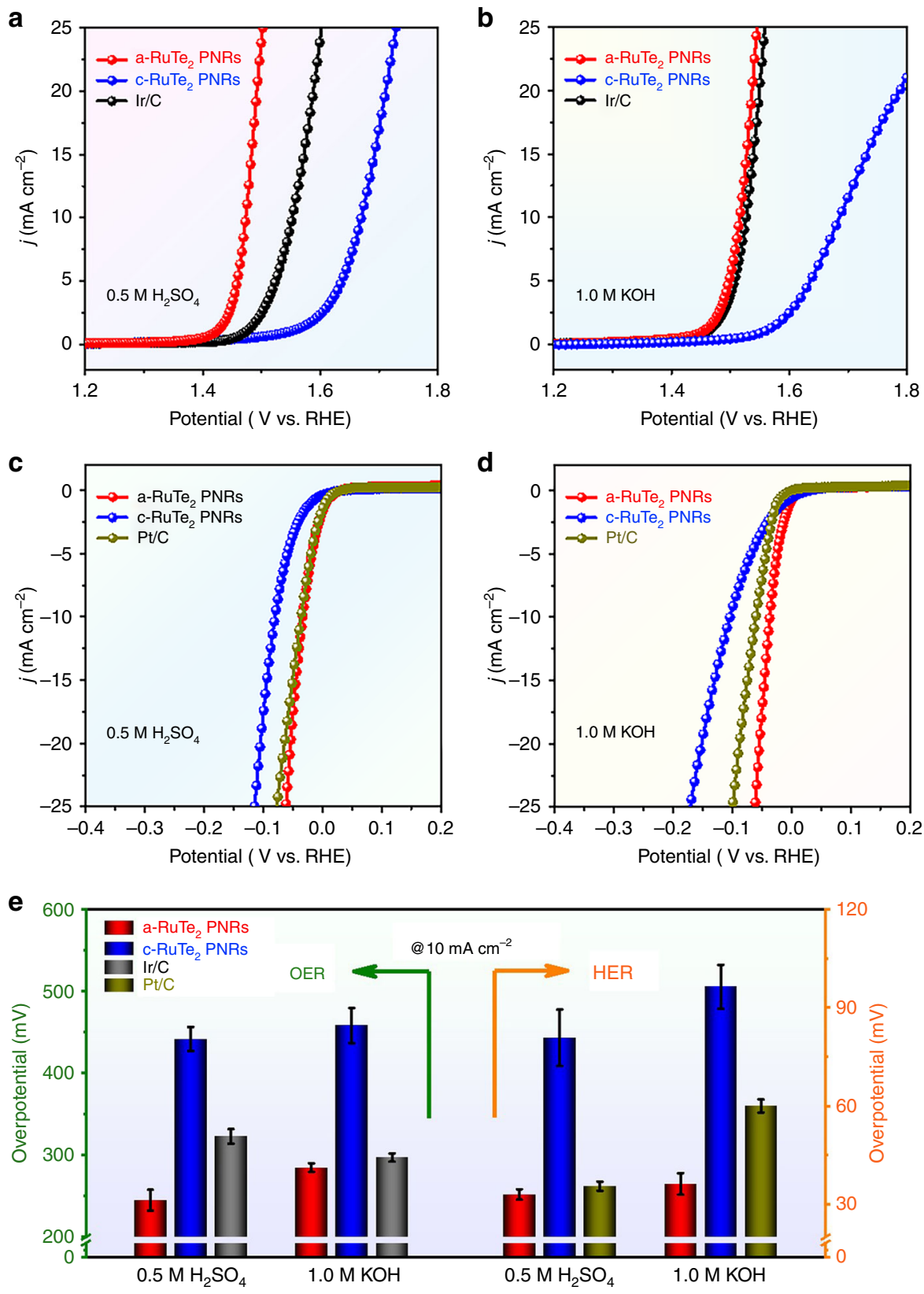

Fig. 6 Electrochemical studies of catalysts in different electrolytes. OER polarization curves of a-RuTe $e_{2}$ PNRs, c-RuTe $e_{2}$ PNRs and commercial Ir/C in a $0.5 \mathrm{M} \mathrm{H}_{2} \mathrm{SO}_{4}$ and $\mathbf{b} 1.0 \mathrm{M} \mathrm{KOH}$. HER polarization curves of a-RuTe 2 PNRs, c-RuTe $\mathrm{PNRs}_{2}$ and commercial Pt/C in c $0.5 \mathrm{M} \mathrm{H} \mathrm{H}_{2} \mathrm{SO}_{4}$ and $\mathbf{d} 1.0 \mathrm{M} \mathrm{KOH}$. e Histogram of overpotentials at $10 \mathrm{~mA} \mathrm{~cm}^{-2}$ from a-d.

alkaline media but also exhibit outstanding activities in $0.05 \mathrm{M}$ $\mathrm{H}_{2} \mathrm{SO}_{4}$ and $0.1 \mathrm{M} \mathrm{KOH}$ (Supplementary Fig. 7). Additionally, the HER activities of various catalysts were investigated. We can see that the HER performance of a-RuTe ${ }_{2}$ PNRs is significantly better than that of $\mathrm{c}-\mathrm{RuTe}_{2}$ PNRs, and even exceed that of $\mathrm{Pt} / \mathrm{C}$ as the applied voltage increasing (Fig. $6 \mathrm{c}$ and Supplementary Table 2). Although all catalysts show similar catalytic activity in $1.0 \mathrm{M}$ $\mathrm{KOH}$ at the beginning, the overpotential of a-RuTe $\mathrm{R}_{2}$ PNR is markedly lower than those of $\mathrm{c}-\mathrm{RuTe}_{2} \mathrm{PNRs}$ and $\mathrm{Pt} / \mathrm{C}$ with increasing voltage (Fig. 6d). Similarly, robust HER activity in $0.05 \mathrm{M} \mathrm{H}_{2} \mathrm{SO}_{4}$ and $0.1 \mathrm{M} \mathrm{KOH}$ can also be observed for a-RuTe PNRs (Supplementary Fig. 8). To further analyze the electrocatalytic activity of various catalysts, we summarize the overpotential at a current density of $10 \mathrm{~mA} \mathrm{~cm}^{-2}$ in different electrolytes (Fig. 6e). Evidently, the OER and HER properties of a-RuTe $e_{2}$ PNRs are superior to those of $c-\mathrm{RuTe}_{2} \mathrm{PNRs}, \mathrm{Ir} / \mathrm{C}$ and
$\mathrm{Pt} / \mathrm{C}$, especially in the acidic environment. Tafel slopes of various catalysts were also summarized in Supplementary Figs. 9, 10, in which the a-RuTe $e_{2}$ PNRs show the smallest Tafel slope in different $\mathrm{pH}$ ranges, representing the fastest reaction kinetics. To evaluate the number of active sites, the copper underpotential deposition ( $\mathrm{Cu}$ UPD) method was performed ${ }^{35,36}$. Compared with c-RuTe ${ }_{2}$ PNRs, an increase in the number of active sites can be observed for a-RuTe 2 PNRs, and thus exhibiting improved catalytic activity (Supplementary Fig. 11). Cyclic voltammetry curves were performed in Supplementary Fig. 12, no redox peaks appeared for a-RuTe $\mathrm{T}_{2}$ PNRs during OER process, demonstrating that their self-oxidation is negligible. Faraday efficiency (FE) of OER and HER are more than 95\%, indicating that the high currents are almost entirely originated from the process of water splitting (Supplementary Fig. 13). In addition, the HER and OER polarization curves of $\mathrm{a}-\mathrm{RuTe}_{2}$ without $\mathrm{iR}$ correction are also 
a

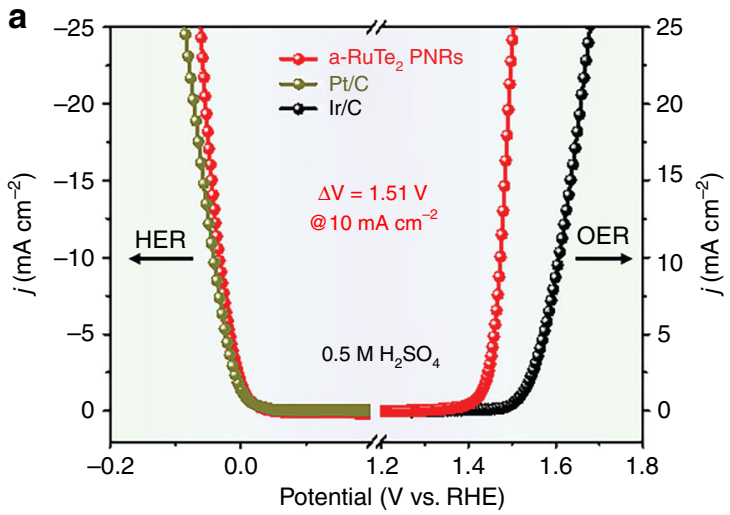

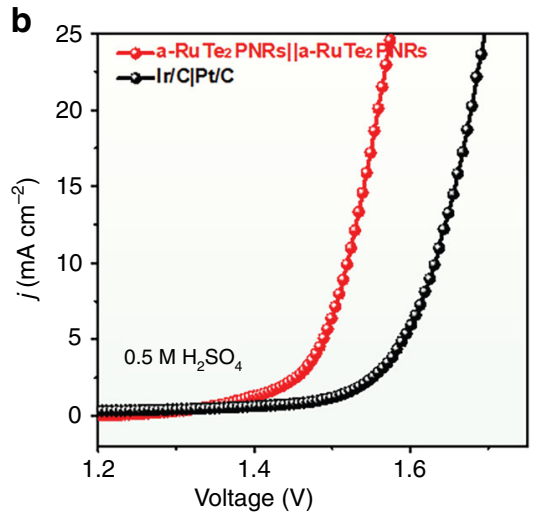

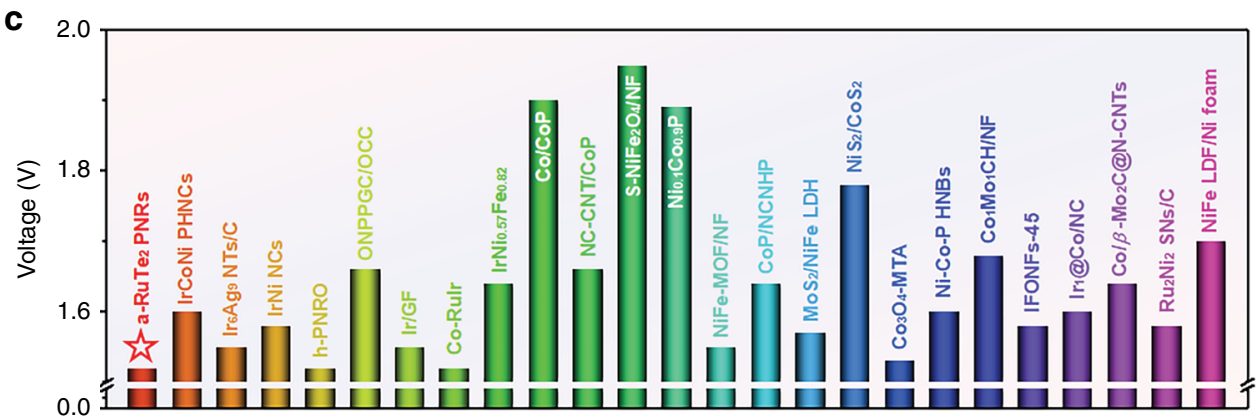

Samples

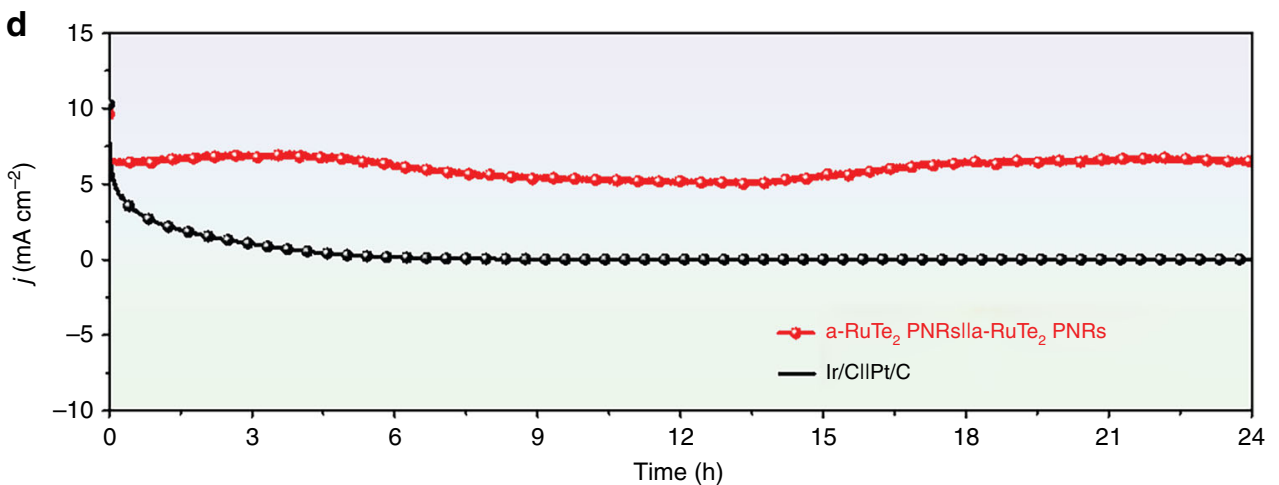

Fig. 7 Overall water splitting studies of catalysts in acidic media. a Polarization curves of a-RuTe $\mathrm{PNRs}$, commercial $\mathrm{Pt} / \mathrm{C}$ and $\mathrm{Ir} / \mathrm{C}$ in $0.5 \mathrm{M} \mathrm{H} \mathrm{HO}_{4}$ for HER and OER. b Polarization curves of a-RuTe 2 PNRs $\|$ a-RuTe ${ }_{2}$ PNRs and $\mathrm{Ir} / \mathrm{C} \| \mathrm{Pt} / \mathrm{C}$ in $0.5 \mathrm{M} \mathrm{H}_{2} \mathrm{SO}_{4}$ for water splitting. c Comparison of the overpotential of a-RuTe $e_{2}$ PNRs and recently reported overall water splitting electrocatalysts at $10 \mathrm{~mA} \mathrm{~cm}{ }^{-2}$. d Time-dependent current density curves of a-RuTe $\mathrm{P}_{2}$ PNRs $\|$ a-RuTe $\mathrm{T}_{2}$ PNRs and $\mathrm{Ir} / \mathrm{C} \| \mathrm{Pt} / \mathrm{C}$ for water splitting in $0.5 \mathrm{M} \mathrm{H}_{2} \mathrm{SO}_{4}$.

performed as reference (Supplementary Fig. 14), where the a$\mathrm{RuTe}_{2}$ PNRs exhibit excellent OER and HER activities under different conditions.

Considering the superior HER and OER activities of a-RuTe PNRs in acidic condition, a two-electrode device for acidic overall water splitting was constructed. Figure 7 a displays the polarization curves of a-RuTe 2 PNRs, Pt/C and $\mathrm{Ir} / \mathrm{C}$ in $0.5 \mathrm{M} \mathrm{H}_{2} \mathrm{SO}_{4}$. At left, the a-RuTe $e_{2}$ PNRs exhibit a slightly improved HER activity compared with $\mathrm{Pt} / \mathrm{C}$. However, at right, the OER activity of a$\mathrm{RuTe}_{2}$ PNRs is significantly better than that of Ir/C. The voltage difference $(\Delta \mathrm{V})$ between HER and OER at the current density of $10 \mathrm{~mA} \mathrm{~cm}^{-2}$ is $1.51 \mathrm{~V}$. When a-RuTe 2 PNRs were employed as both anode and cathode catalysts in $0.5 \mathrm{M} \mathrm{H}_{2} \mathrm{SO}_{4}$, only a cell voltage of $1.52 \mathrm{~V}$ is needed to achieve $10 \mathrm{~mA} \mathrm{~cm}^{-2}$, which is much better than that of $\mathrm{Ir} / \mathrm{C} \| \mathrm{Pt} / \mathrm{C}$ (Fig. 7b). Most importantly, the cell voltage of a-RuTe $\mathrm{RNR}_{2} \mathrm{PN}-\mathrm{RuTe}_{2} \mathrm{PNRs}$ for water splitting at $10 \mathrm{~mA} \mathrm{~cm}^{-2}$ even surpasses most reported electrocatalysts (Fig. 7c and Supplementary Table 3). The chronoamperometry curve was then carried out to evaluate the long-term stability of a-RuTe $e_{2}$ PNRs $\| \mathrm{a}-\mathrm{RuTe}_{2}$ PNRs (Fig. 7d). Compared with $\mathrm{Ir} / \mathrm{C} \| \mathrm{Pt} / \mathrm{C}$, the a-RuTe $\mathrm{P}_{2}$ PNRs $\| \mathrm{a}-\mathrm{RuTe}_{2}$ PNRs exhibit improved stability in acidic condition $(24 \mathrm{~h})$. Detailed characterizations were carried out to further understand the enhanced activity and stability of a-RuTe $e_{2}$ PNRs for water splitting in acidic conditions (Supplementary Fig. 15, 16). We can see that the porous morphology and bullet-like outline were largely maintained while $\mathrm{Ir} / \mathrm{C}$ and $\mathrm{Pt} / \mathrm{C}$ were severely agglomerated (Supplementary Figs. 17, 18). The amorphous feature of a-RuTe $\mathrm{R}_{2}$ PNRs can also be largely maintained. Additionally, the a-RuTe ${ }_{2}$ PNRs can maintain excellent chemical stability even under 5.0 $\mathrm{M} \mathrm{H}_{2} \mathrm{SO}_{4}$ at $60^{\circ} \mathrm{C}$ for $1 \mathrm{~h}$ (Supplementary Fig. 19). Therefore, the a-RuTe PNRs not only exhibit excellent chemical corrosion resistance but also present superior electrochemical stability.

Mechanistic investigations. To further explore the reasons for the significant activity difference of a-RuTe $e_{2}$ PNRs and c$\mathrm{RuTe}_{2}$ PNRs, electrochemically active surface area (ECSA) was 

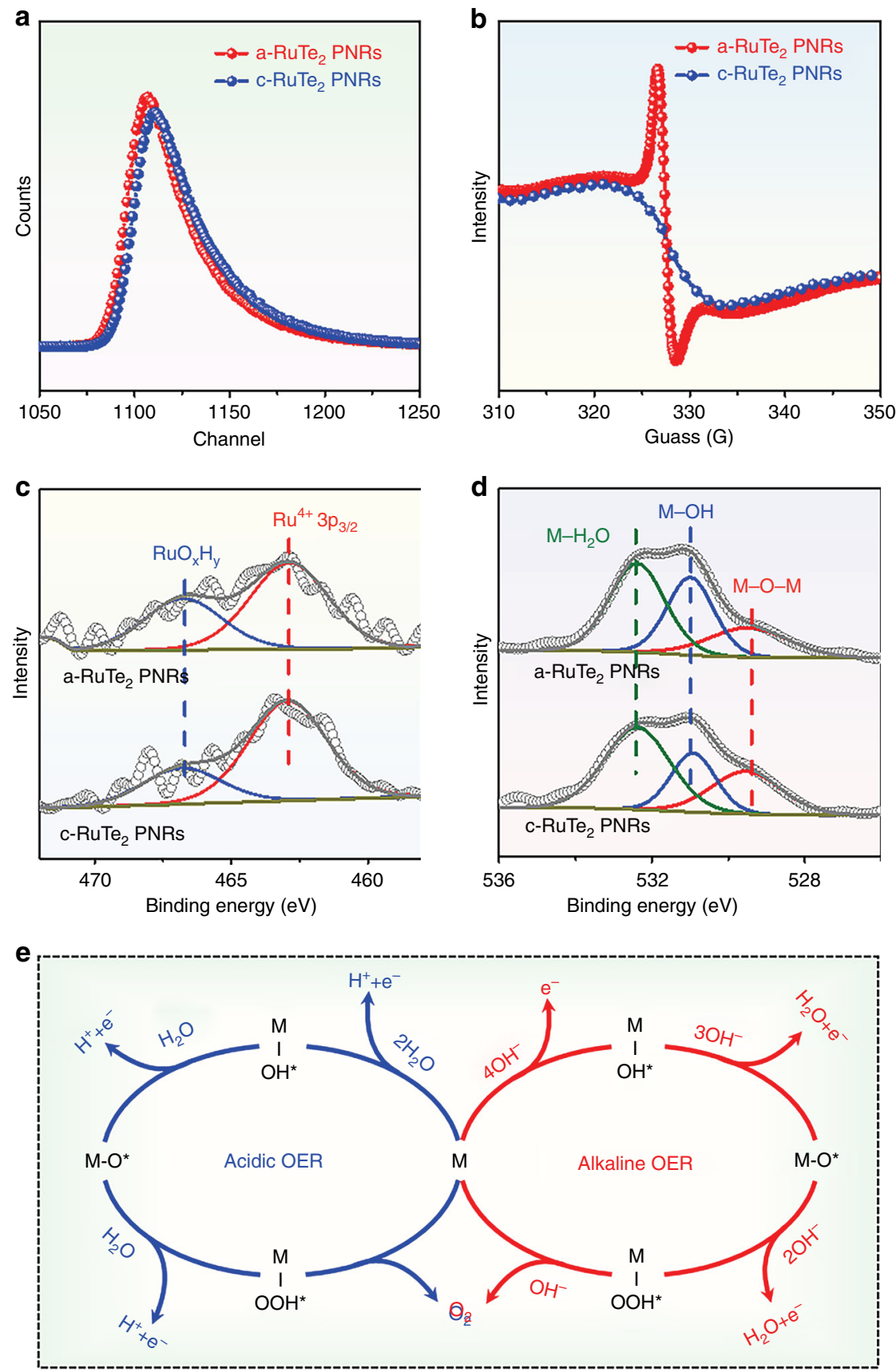

Fig. 8 Mechanism investigations. a Positron lifetime spectra, b ESR spectra, c Ru 3p, and d O 1s XPS spectra of a-RuTe $e_{2} P_{N R s}$ and c-RuTe 2 PNRs. e Schematic illustration of the OER process under acidic and alkaline conditions.

calculated (Supplementary Fig. 20 and Supplementary Table 4). Apparently, an improved ECSA that represents more exposed active sites can be observed for a-RuTe 2 PNRs, which may be caused by the distortions of $\mathrm{Ru}-\mathrm{Te}$ bond that derived from the high density of defects in amorphous materials. This finding will also verify the previous theoretical investigations on the local electronic structures. Positron annihilation spectroscopy (PAS) and corresponding lifetime parameters were then performed (Fig. 8a and Supplementary Table 5). We can see that the a-RuTe PNRs exhibit a significantly prolonged lifetime $\left(\tau_{1}\right.$ and $\left.\tau_{2}\right)$ than their crystalline counterparts, mainly due to high density of defects that changed the electron density. To prove this conjecture, electron spin resonance (ESR) measurement was also carried to probe the unpaired electrons that generated by defects
(Fig. 8b), in which a pair of sharp peaks can be clearly observed for a-RuTe ${ }_{2}$ PNRs when compared with $\mathrm{c}-\mathrm{RuTe}_{2}$ PNRs, supporting the presence of a high density of defects in the a-RuTe PNRs. In fact, the presence of defective sites can not only affect the electronic distribution caused by the distortion of $\mathrm{Ru}-\mathrm{Te}$ bond but also combine with oxygen atoms to form $\mathrm{RuO}_{\mathrm{x}} \mathrm{H}_{\mathrm{y}}$ species which are conducive the reaction process ${ }^{37}$. Therefore, X-ray photoelectron spectroscopy (XPS) was employed to measure the chemical composition and electronic configuration. As shown in Fig. 8c, compared with c-RuTe $\mathrm{C}_{2}$ PNRs, a significantly enhanced peak area of $\mathrm{RuO}_{\mathrm{x}} \mathrm{H}_{\mathrm{y}}$ species can be observed for a-RuTe$e_{2}$ PNRs, indicating that the presence of defects was filled with oxygen and then to form $\mathrm{OH}$ species. The increased $\mathrm{OH}$ species in $\mathrm{a}-\mathrm{RuTe}_{2}$ PNRs can also be demonstrated by O 1s XPS (Fig. 8d), in which 
the relative peak area of $\mathrm{M}-\mathrm{OH}$ species increase, whereas the $\mathrm{M}$ $\mathrm{O}-\mathrm{M}$ species decrease. In view of the experimental results and theoretical analysis, possible OER mechanisms under $\mathrm{pH}$ universal conditions have been depicted in Fig. 8e. As illustrated, for acidic OER process, water molecule preferentially splitting on the catalyst's surface and then gradually dehydrogenate to form $\mathrm{M}-\mathrm{OOH}^{*}$ intermediate. Finally, $\mathrm{O}_{2}$ is successfully released directly from the surface and the active site continues to proceed with the catalytic reaction. Under the alkaline condition, the dissociated $\mathrm{OH}^{-}$is adsorbed on the catalyst's surface and then form $\mathrm{M}-\mathrm{OH}^{*}$ intermediate. Subsequently, the $\mathrm{M}-\mathrm{OH}^{*}$ combines with $\mathrm{OH}^{-}$to release water molecule and then becomes $\mathrm{M}-\mathrm{O}^{*}$. The metastable $\mathrm{M}-\mathrm{O}^{*}$ intermediate will soon combine with $\mathrm{OH}^{-}$to generate $\mathrm{M}-\mathrm{OOH}^{*}$ and eventually releases $\mathrm{O}_{2}$.

\section{Discussion}

In summary, theoretical calculations have supplied an insightful understanding of the intrinsic high electroactivity that directionally guide the experimental synthesis of the a- $\mathrm{RuTe}_{2}$ PNRs as the efficient water splitting electrocatalysts. DFT calculations reveal that the local flexible Te-bonding configurations are yielded from a strong $\mathrm{p}-\mathrm{d}$ transfer induced $\mathrm{p}-\pi$ sensitivity enhancement, which renders the stabilization of distortion-strain as well as elevates electronic activities near the Fermi level through an effective annihilation of the crystal-field-splitting effect of Rusites. Within this trend, the local distorted Ru-Te lattice increases the homogeneity for efficient inter-d-orbital electron-transfer ability among Ru sites. Therefore, the short-range disorder promotes the electron-lattice coupling effect but also boosts OER catalysis within $\mathrm{pH}$-universal conditions. As a result, the a- $\mathrm{RuTe}_{2}$ PNRs exhibit superior HER and OER activities than those of their crystalline counterparts. More importantly, a relatively low cell potential of $1.52 \mathrm{~V}$ has been achieved for reaching the current density of $10 \mathrm{~mA} \mathrm{~cm}^{-2}$ in water splitting, representing much enhanced activity under acidic conditions. Detailed investigations show that the generation of distorted Ru-Te bonds is attributed to the extensive defects in the amorphous structure. These defects will be substituted by oxygen atoms to form $\mathrm{RuO}_{\mathrm{x}} \mathrm{H}_{\mathrm{y}}$ species that will promote the catalytic activity. Our work provides a feasible strategy in amorphous catalysts design and investigation that offers valuable insight to the development of a new generation of catalysts, which will broaden the horizon of future electrocatalyst studies in energy applications.

\section{Materials and methods}

Chemicals. Potassium tellurite $\left(\mathrm{K}_{2} \mathrm{TeO}_{3}, 99.5 \%\right)$ was purchased from Aladdinreagent Inc. Hexaammineruthenium (III) chloride $\left(\mathrm{Cl}_{3} \mathrm{H}_{18} \mathrm{~N}_{6} \mathrm{Ru}, \mathrm{Ru} 32.1 \%\right)$ was purchased from Alfa Aesar. Poly (vinylpyrrolidone) (PVP, average M.W. 58000, K15-19) was purchased from J\&K Scientific Ltd. Ammonia solution $\left(\mathrm{NH}_{3} \cdot \mathrm{H}_{2} \mathrm{O}\right.$, AR), hydrazine hydrate aqueous solution $\left(\mathrm{N}_{2} \mathrm{H}_{4} \cdot \mathrm{H}_{2} \mathrm{O}, \mathrm{AR}\right)$ and isopropanol (IPA, AR) were purchased from Sinopharm Chemical Reagent Co., Ltd. Argon (Ar, 99.999\%) was purchased from WuGang Gas Co., Ltd. (Shanghai, China). Pt/C (20 wt\% Pt on Vulcan black) was purchased from Shanghai Hesen Electric Co., Ltd. Ir/C (20 wt\% Ir on Vulcan black) was from Premetek Co., Ltd.

Synthesis of $\mathbf{R u T e}_{2}$ PNRs. $3.6 \mathrm{mg} \mathrm{Cl}{ }_{3} \mathrm{H}_{18} \mathrm{~N}_{6} \mathrm{Ru}(11.6 \mu \mathrm{mol}), 6.0 \mathrm{mg} \mathrm{K}_{2} \mathrm{TeO}_{3}$ $(23.6 \mu \mathrm{mol})$ and $65.0 \mathrm{mg}$ PVP $(1.1 \mu \mathrm{mol})$ were dissolved in $2 \mathrm{~mL} \mathrm{H}_{2} \mathrm{O}$. After a few minutes of sonication, $2.0 \mathrm{~mL} \mathrm{NH} \cdot \mathrm{H}_{2} \mathrm{O}$ and $1.0 \mathrm{~mL} \mathrm{~N} \mathrm{H}_{4} \cdot \mathrm{H}_{2} \mathrm{O}$ were quickly injected into the above mixture. The mixture solution was then transferred into Teflon-sealed autoclave and maintained at $180^{\circ} \mathrm{C}$ for $3 \mathrm{~h}$. The $\mathrm{RuTe}_{2}$ PNRs were obtained by washed several times with ethanol/acetone solution.

Synthesis of a-RuTe $\mathbf{2}_{\mathbf{2}}$ PNRs and c-RuTe $\mathbf{2}_{\mathbf{2}}$ PNRs. $\mathrm{RuTe}_{2}$ PNRs were deposited on VC-X72 carbon (Ru loading of $20 \mathrm{wt} \%$, determined by ICP-AES) in ethanol solution by sonicating for $30 \mathrm{~min}$. The resulting products were separated by centrifugation and washed several times using ethanol/acetone solution. The products were annealed at $250^{\circ} \mathrm{C}$ in air for $5 \mathrm{~h}$ to yield a-RuTe ${ }_{2}$ PNRs. The c-RuTe $e_{2}$ PNRs were obtained by annealing treatment at $250{ }^{\circ} \mathrm{C}$ in $\mathrm{Ar}$ for $5 \mathrm{~h}$ and then at $250^{\circ} \mathrm{C}$ in air for $1 \mathrm{~h}$.

Characterization. Low-magnification transmission electron microscopy (TEM) was performed on a HITACHI HT7700 at $120 \mathrm{kV}$. High-angle annular dark-field scanning transmission electron microscopy (HAADF-STEM) and HRTEM was recorded on a FEI Talos F200X S/TEM with a field-emission gun at $200 \mathrm{kV}$. ESR spectra were collected on JEOL JES-X320. XPS was performed on SSI S-Probe XPS Spectrometer. Powder X-ray diffraction (PXRD) patterns were collected on X'PertPro MPD diffractometer (Netherlands PANalytical) with a $\mathrm{Cu} \mathrm{Ka}$ X-ray source $(\lambda=1.540598 \AA)$.

Electrochemical measurements. Electrochemical measurements were performed on CHI660 workstation (Chenhua, Shanghai) by using the three-electrode system. Graphite rod and saturated calomel electrode were used as counter and reference electrode, respectively. To prepare the catalyst ink, $2 \mathrm{mg}$ catalysts were added into a mixture solution including $800 \mu \mathrm{L}$ IPA, $200 \mu \mathrm{L} \mathrm{H}_{2} \mathrm{O}$ and $5 \mu \mathrm{L}$ Nafion. After $30 \mathrm{~min}$ sonication, $20 \mu \mathrm{L}$ catalyst ink was deposited on glassy carbon electrode (diameter $5 \mathrm{~mm}$, area: $0.196 \mathrm{~cm}^{2}$ ) as a working electrode. Polarization curves were then performed in a broad $\mathrm{pH}$ range, including $0.5 \mathrm{M} \mathrm{H}_{2} \mathrm{SO}_{4}, 0.05 \mathrm{M} \mathrm{H}_{2} \mathrm{SO}_{4}, 0.1 \mathrm{M}$ $\mathrm{KOH}$ and $1.0 \mathrm{M} \mathrm{KOH}$, after a continuous cyclic voltammetry. All polarization curves in this study are the average of the stable polarization curves scanned in three experiments. The solution resistance (Rs) is $\sim 6 \Omega$ in $0.5 \mathrm{M} \mathrm{H}_{2} \mathrm{SO}_{4}, \sim 39 \Omega$ in $0.05 \mathrm{M} \mathrm{H}_{2} \mathrm{SO}_{4}, \sim 36 \Omega$ in $0.1 \mathrm{M} \mathrm{KOH}$ and $\sim 5 \Omega$ in $1 \mathrm{M} \mathrm{KOH}$. The Tafel slopes were derived from polarization curves and $95 \%$ iR compensation in all the solutions. Long-term stability for water splitting was tested by using a two-electrode system. The underpotential deposition (UPD) method was used to qualify active sites. The number of active sites can be calculated with the equation: $n=\mathrm{Q}_{\mathrm{Cu}} / 2 \mathrm{~F}$, where $\mathrm{Q}$ is the UPD Cu stripping charge $\left(\mathrm{Q}_{\mathrm{Cu}}, \mathrm{Cu}_{\text {upd }} \rightarrow \mathrm{Cu}^{2+}+2 \mathrm{e}^{-}\right)$and F is the Faraday constant. The Faraday efficiency (FE) measurements were conducted on an $\mathrm{H}$-cell reactor where each chamber of $\sim 60 \mathrm{~mL}$ was filled with $30 \mathrm{~mL}$ of $0.5 \mathrm{M} \mathrm{H}_{2} \mathrm{SO}_{4}$ solution and the two chambers were separated by an anion exchange membrane (Nafion 117). The Ar (30 sccm) was applied throughout the HER and OER measurements. Chronoamperometry measurements were carried out at the voltage that the current density reached $10 \mathrm{~mA} \mathrm{~cm}^{-2}$. The reactor was directly connected to the gas chromatograph (GC Agilent 7890B). The FE of a product was calculated as follows: $\mathrm{FE}=e \mathrm{~F} \times n / \mathrm{Q}$, where $\mathrm{e}$ is the number of electrons transferred of the product, $\mathrm{Q}$ is the total charge in HER and OER process, $\mathrm{n}$ is the number of moles of the product and F is the Faraday constant.

Computational details. Rotationally invariant $\mathrm{DFT}+\mathrm{U}$ calculations within CASTEP code has been performed ${ }^{38,39}$. The algorithm of Broyden-FletcherGoldfarb-Shannon (BFGS) has chosen for all related ground state geometry optimization, especially for the interfacial relaxation. The cutoff energy of plane-wave basis sets for total energy and valence electronic states calculations has been set to $750 \mathrm{eV}$. The PBE exchange-correlation functional is selected for DFT $+\mathrm{U}$ calculations. The applied $\mathrm{U}$ values in this work are 2.38 and $0.36 \mathrm{eV}$ for $\mathrm{Ru}$ and $\mathrm{Te}$, respectively. To improve the convergence quality of the transition metal compound system, the ensemble DFT (EDFT) method of Marzari et al. is used during the electronic-minimization process ${ }^{40}$.

To approach a realistic local short-range ordered structure, a fixed volume NVT ensemble has been used for ab-initio molecular dynamics (AIMD) to conduct an analogue anneal-to-quench process from 1600 to $300 \mathrm{~K}$. All of these AIMD simulations have been performed onto an expanded supercell of crystalline $\mathrm{RuTe}_{2}$ lattice with a density of $6.036 \mathrm{~g} / \mathrm{cm}^{3}\left(8.303 \mathrm{~g} / \mathrm{cm}^{3}\right.$ for crystalline). The original crystalline $\mathrm{RuTe}_{2}$ unit-cell lattice has been imported from the database with group symmetry of PNNM and experimental lattice parameters $(a=5.38 \AA, b=6.49 \AA$, and $c=4.08 \AA$ ). The geometry optimization is also applied to the randomly selective trajectories of the MD process. The weighted average coordination $(\mathrm{CN})$ of $\mathrm{Ru}$ is lowered ( $\mathrm{CN}=6$ for crystalline $\mathrm{RuTe}_{2}$ ) staying between 4 (tetrahedral) and 6 (octahedral), while the Te sites are more flexible ranged from $\mathrm{CN}=2$ to $\mathrm{CN}=6$ containing Te-Te bonds. Considering the DFT computational cost, the MonkhostPack reciprocal space integration was performed using Gamma-center-off special k-points with a mesh of $2 \times 2 \times 2$, which was guided by the initial convergence test $^{41}$. With these settings, the overall total energy for each step is converged to less than $5.0 \times 10^{-7} \mathrm{eV}$ per atom. The Hellmann-Feynman forces on the atom were converged to less than $0.001 \mathrm{eV} / \AA ̊$.

$\mathrm{The} \mathrm{Ru}, \mathrm{Te}, \mathrm{O}$, and $\mathrm{H}$ norm-conserving pseudopotentials are generated using the OPIUM code in the Kleinman-Bylander projector form, and the non-linear partial core correction and a scalar relativistic averaging scheme are used to treat the mixed valence Co spin-orbital coupling effect ${ }^{42-44}$. We chose the projectorbased $(4 d, 5 s, 5 p),(5 s, 5 p),(2 s, 2 p)$, and (1s) states to reflect the valence states of Ru, $\mathrm{Te}, \mathrm{O}$, and $\mathrm{H}$ atoms, respectively. The RRKJ method is chosen for the optimization of the pseudopotentials ${ }^{45}$

To achieve the mass and electron conservation, the key adsorbates and coreactants are both considered in the calculation of the energetic diagram for the water splitting reactions. For each intermediate, we have fully considered the possible diffusions on the surface to locate the most stable adsorption site and 
corresponding coordination number $(\mathrm{CN})$ environment in the complicated amorphous structure modelling.

For the acidic OER, the acidic OER reactions are as below.

$$
\begin{gathered}
2 \mathrm{H}_{2} \mathrm{O}^{*} \rightarrow \mathrm{OH}^{*}+\mathrm{H}_{2} \mathrm{O}+\left(\mathrm{H}^{+}+\mathrm{e}^{-}\right) \\
\mathrm{OH}^{*}+\mathrm{H}_{2} \mathrm{O}+\left(\mathrm{H}^{+}+\mathrm{e}^{-}\right) \rightarrow \mathrm{O}^{*}+\mathrm{H}_{2} \mathrm{O}+\left(2 \mathrm{H}^{+}+2 \mathrm{e}^{-}\right) \\
\mathrm{O}^{*}+\mathrm{H}_{2} \mathrm{O}+\left(2 \mathrm{H}^{+}+2 \mathrm{e}^{-}\right) \rightarrow \mathrm{OOH}^{*}+\left(3 \mathrm{H}^{+}+3 \mathrm{e}^{-}\right) \\
\mathrm{OOH}^{*}+\left(3 \mathrm{H}^{+}+3 \mathrm{e}^{-}\right) \rightarrow \mathrm{O}_{2}(\mathrm{~g})+\left(4 \mathrm{H}^{+}+4 \mathrm{e}^{-}\right)
\end{gathered}
$$

On the contrary, the OER process in alkaline medium renders reactions are as below.

$$
\begin{gathered}
4 \mathrm{OH}^{-} \rightarrow \mathrm{OH}^{*}+3 \mathrm{OH}^{-}+\mathrm{e}^{-} \\
\mathrm{OH}^{*}+3 \mathrm{OH}^{-}+\mathrm{e}^{-} \rightarrow \mathrm{O}^{*}+2 \mathrm{OH}^{-}+\mathrm{H}_{2} \mathrm{O}+2 \mathrm{e}^{-} \\
\mathrm{O}^{*}+2 \mathrm{OH}^{-}+\mathrm{H}_{2} \mathrm{O}+2 \mathrm{e}^{-} \rightarrow{ }^{*} \mathrm{OOH}+\mathrm{OH}^{-}+2 \mathrm{H}_{2} \mathrm{O}+3 \mathrm{e}^{-} \\
* \mathrm{OOH}+\mathrm{OH}^{-}+2 \mathrm{H}_{2} \mathrm{O}+3 \mathrm{e}^{-} \rightarrow \mathrm{O}_{2}+2 \mathrm{H}_{2} \mathrm{O}+4 \mathrm{e}^{-}
\end{gathered}
$$

\section{Data availability}

The data that support the findings of this study are available from the corresponding author upon request.

Received: 2 July 2019; Accepted: 11 November 2019; Published online: 12 December 2019

\section{References}

1. Zang, Y. et al. Tuning orbital orientation endows molybdenum disulfide with exceptional alkaline hydrogen evolution capability. Nat. Commun. 10, 1217 (2019).

2. Wei, C. et al. Approaches for measuring the surface areas of metal oxide electrocatalysts for determining their intrinsic electrocatalytic activity. Chem. Soc. Rev. 48, 2518-2534 (2019).

3. An, L. et al. A self-standing high-performance hydrogen evolution electrode with nanostructured $\mathrm{NiCO}_{2} \mathrm{O}_{4} / \mathrm{CuS}$ heterostructures. Adv. Funct. Mater. 25, 6814-6822 (2015).

4. Zhang, L., Xiao, J., Wang, H. \& Shao, M. Carbon-based electrocatalysts for hydrogen and oxygen evolution reactions. ACS Catal. 7, 7855-7865 (2017).

5. $\mathrm{Du}, \mathrm{N}$. et al. Trimetallic tristar nanostructures: tuning electronic and surface structures for enhanced electrocatalytic hydrogen evolution. Adv. Mater. 28, 2077-2084 (2016).

6. Bai, S. et al. Surface polarization matters: enhancing the hydrogen-evolution reaction by shrinking Pt shells in Pt-Pd-graphene stack structures. Angew. Chem. Int. Ed. 53, 12120-12124 (2014).

7. Seitz, L. C. et al. A highly active and stable $\mathrm{IrOx} / \mathrm{SrIrO}_{3}$ catalyst for the oxygen evolution reaction. Science 353, 1011-1014 (2016).

8. Hurst, J. K. In pursuit of water oxidation catalysts for solar fuel production. Science 328, 315-316 (2010).

9. Jiao, Y., Zheng, Y., Jaroniec, M. \& Qiao, S. Z. Design of electrocatalysts for oxygen- and hydrogen-involving energy conversion reactions. Chem. Soc. Rev. 44, 2060-2086 (2015).

10. Ye, S. H., Shi, Z. X., Feng, J. X., Tong, Y. X. \& Li, G. R. Activating $\mathrm{CoOOH}$ porous nanosheet arrays by partial iron substitution for efficient oxygen evolution reaction. Angew. Chem. Int. Ed. 57, 2672-2676 (2018).

11. Sun, S. et al. Shifting oxygen charge towards octahedral metal: a way to promote water oxidation on cobalt spinel oxides. Angew. Chem. Int. Ed. 131, 6103-6108 (2019).

12. Wang, J. et al. In situ formation of molecular Ni-Fe active sites on heteroatomdoped graphene as a heterogeneous electrocatalyst toward oxygen evolution. Sci. Adv. 4, eaap7970 (2018).

13. Zhao, X. et al. Engineering the electrical conductivity of lamellar silver-doped cobalt(II) selenide nanobelts for enhanced oxygen evolution. Angew. Chem. Int. Ed. 56, 328-332 (2017).

14. Xu, H., Shi, Z. X., Tong, Y. X. \& Li, G. R. Porous microrod arrays constructed by carbon-confined $\mathrm{NiCo@NiCoO}$ core@shell nanoparticles as efficient electrocatalysts for oxygen evolution. Adv. Mater. 30, 1705442 (2018).

15. Zhu, H. et al. When cubic cobalt sulfide meets layered molybdenum disulfide: a core-shell system toward synergetic electrocatalytic water splitting. $A d v$. Mater. 27, 4752-4759 (2015).
16. Wang, X. et al. Plasma-triggered synergy of exfoliation, phase transformation, and surface engineering in cobalt diselenide for enhanced water oxidation. Angew. Chem. Int. Ed. 130, 16659-16663 (2018).

17. Jia, Y. et al. A heterostructure coupling of exfoliated Ni-Fe hydroxide nanosheet and defective graphene as a bifunctional electrocatalyst for overall water splitting. Adv. Mater. 29, 1700017 (2017).

18. Feng, J. et al. Iridium-based multimetallic porous hollow nanocrystals for efficient overall-water-splitting catalysis. Adv. Mater. 29, 1703798 (2017).

19. Wei, C. et al. Recommended practices and benchmark activity for hydrogen and oxygen electrocatalysis in water splitting and fuel cells. Adv. Mater. 31, 1806296 (2019).

20. Zheng, Y. et al. Understanding the roadmap for electrochemical reduction of $\mathrm{CO}_{2}$ to multi-carbon oxygenates and hydrocarbons on copper-based catalysts. J. Am. Chem. Soc. 141, 7646-7659 (2019).

21. Shan, J. et al. Charge-redistribution-enhanced nanocrystalline $\mathrm{Ru} @ \mathrm{IrO}_{\mathrm{x}}$ electrocatalysts for oxygen evolution in acidic media. Chem 5, 445-459 (2019).

22. Wang, P. et al. Precise tuning in platinum-nickel/nickel sulfide interface nanowires for synergistic hydrogen evolution catalysis. Nat. Commun. 8 , 14580 (2017)

23. Lu, Q. et al. Crystal phase-based epitaxial growth of hybrid noble metal nanostructures on $4 \mathrm{H} / \mathrm{fcc}$ Au nanowires. Nat. Chem. 10, 456-461 (2018).

24. Wang, J. et al. Phase and structure modulating of bimetallic CuSn nanowires boosts electrocatalytic conversion of $\mathrm{CO}_{2}$. Nano Energy 59, 138-145 (2019).

25. Nsanzimana, J. M. V. et al. An efficient and earth-abundant oxygen-evolving electrocatalyst based on amorphous metal borides. Adv. Energy Mater. 8, 1701475 (2017).

26. Sun, J. et al. Sandwich-like reduced graphene oxide/carbon black/amorphous cobalt borate nanocomposites as bifunctional cathode electrocatalyst in rechargeable zinc-air batteries. Adv. Energy Mater. 8, 1801495 (2017).

27. Yang, N. et al. Novel amorphous/crystalline hetero-phase Pd nanosheets: onepot synthesis and highly selective hydrogenation reaction. Adv. Mater. 30, 1803234 (2018).

28. Zhang, J., Yin, R., Shao, Q., Zhu, T. \& Huang, X. Oxygen vacancies in amorphous $\mathrm{InO}_{\mathrm{x}}$ nanoribbons enhance $\mathrm{CO}_{2}$ adsorption and activation for $\mathrm{CO}_{2}$ electroreduction. Angew. Chem. Int. Ed. 58, 5609-5613 (2019).

29. Lu, Y., Wang, J., Peng, Y., Fisher, A. \& Wang, X. Highly efficient and durable $\mathrm{Pd}$ hydride nanocubes embedded in $2 \mathrm{D}$ amorphous $\mathrm{NiB}$ nanosheets for oxygen reduction reaction. Adv. Energy Mater. 7, 1700919 (2017).

30. Zhang, $\mathrm{X}$. et al. Lithiation-induced amorphization of $\mathrm{Pd}_{3} \mathrm{P}_{2} \mathrm{~S}_{8}$ for highly efficient hydrogen evolution. Nat. Cat. 1, 460-468 (2018).

31. Gao, Q. et al. Phase-selective syntheses of cobalt telluride nanofleeces for efficient oxygen evolution catalysts. Angew. Chem. Int. Ed. 56, 7769-7773 (2017).

32. Sun, J. et al. Assembling ultrasmall copper-doped ruthenium oxide nanocrystals into hollow porous polyhedra: highly robust electrocatalysts for oxygen evolution in acidic media. Adv. Mater. 30, 1801351 (2018)

33. Yao, Q. et al. Channel rich $\mathrm{RuCu}$ nanosheets for $\mathrm{pH}$-universal overall water splitting electrocatalysis. Angew. Chem. Int. Ed. 58, 13983-13988 (2019).

34. Gong, Q. et al. Highly stable and methanol tolerant $\mathrm{RuTe}_{2} / \mathrm{C}$ electrocatalysts in alkaline media. ECS Trans. 80, 1023-1032 (2017).

35. Mahmood, J. et al. An efficient and $\mathrm{pH}$-universal ruthenium-based catalyst for the hydrogen evolution reaction. Nat. Nanotech. 12, 441-446 (2017).

36. Zheng, Y. et al. High electrocatalytic hydrogen evolution activity of an anomalous ruthenium catalyst. J. Am. Chem. Soc. 138, 16174-16181 (2016).

37. Reier, T. et al. Molecular insight in structure and activity of highly efficient, low-Ir Ir-Ni oxide catalysts for electrochemical water splitting (OER). J. Am. Chem. Soc. 137, 13031-13040 (2015).

38. Anisimov, V. I., Aryasetiawan, F. \& Lichtenstein, A. I. First-principles calculations of the electronic structure and spectra of strongly correlated systems: the LDA+ U method. J. Phys. Condens. Matter 9, 767-808 (1997).

39. Clark, S. J. et al. First principles methods using CASTEP. Z. Kristallogr. 220, 567-570 (2005)

40. Marzari, N., Vanderbilt, D. \& Payne, M. C. Ensemble density-functional theory for $\mathrm{Ab}$ initio molecular dynamics of metals and finite-temperature insulators. Phys. Rev. Lett. 79, 1337-1340 (1997).

41. Probert, M. I. J. \& Payne, M. C. Improving the convergence of defect calculations in supercells: An ab initio study of the neutral silicon vacancy. Phys. Rev. B 67, 075204 (2003).

42. Kleinman, L. \& Bylander, D. M. Efficacious form for model pseudopotentials. Phys. Rev. Lett. 48, 1425-1428 (1982).

43. Louie, S. G., Froyen, S. \& Cohen, M. L. Nonlinear ionic pseudopotentials in spin-density-functional calculations. Phys. Rev. B 26, 1738-1742 (1982).

44. Grinberg, I., Ramer, N. J. \& Rappe, A. M. Transferable relativistic Dirac-Slater pseudopotentials. Phys. Rev. B 62, 2311-2314 (2000).

45. Rappe, A. M., Rabe, K. M., Kaxiras, E. \& Joannopoulos, J. D. Optimized pseudopotentials. Phys. Rev. B 41, 1227-1230 (1990). 


\section{Acknowledgements}

We thank the financial supports by the National Natural Science Foundation of China $(21571135,2177156)$, the Early Career Start-up fund (PolyU 253026/16P) from the Research Grant Committee (RGC) in Hong Kong, the Ministry of Science and Technology of China (2016YFA0204100, 2017YFA0208200), Young Thousand Talented Program, the Natural Science Foundation of Jiangsu Higher Education Institutions (17KJB150032), the project of scientific and technologic infrastructure of Suzhou (SZS201708), the Priority Academic Program Development of Jiangsu Higher Education Institutions (PAPD), and start-up support from Soochow University.

\section{Author contributions}

X.H. conceived and supervised the research. X.H. and J.W. designed the experiments. X.H., J.W., L.H., Q.S. and H.X. performed most of the experiments and data analysis. X.H., J.W. L.H., H.X. and Q.S. participated in various aspects of the experiments and discussions. B.H. performed the DFT simulations and theoretically guided the overall experimental research design with in-depth mechanism interpretations. X.H., J.W. and B.H. wrote the paper. All authors discussed the results and commented on the paper.

\section{Competing interests}

The authors declare no competing interests.

\section{Additional information}

Supplementary information is available for this paper at https://doi.org/10.1038/s41467019-13519-1.
Correspondence and requests for materials should be addressed to B.H. or X.H.

Peer review information Nature Communications thanks Ivano Castelli and the other, anonymous, reviewer(s) for their contribution to the peer review of this work.

Reprints and permission information is available at http://www.nature.com/reprints

Publisher's note Springer Nature remains neutral with regard to jurisdictional claims in published maps and institutional affiliations.

(c) (i) Open Access This article is licensed under a Creative Commons Attribution 4.0 International License, which permits use, sharing, adaptation, distribution and reproduction in any medium or format, as long as you give appropriate credit to the original author(s) and the source, provide a link to the Creative Commons license, and indicate if changes were made. The images or other third party material in this article are included in the article's Creative Commons license, unless indicated otherwise in a credit line to the material. If material is not included in the article's Creative Commons license and your intended use is not permitted by statutory regulation or exceeds the permitted use, you will need to obtain permission directly from the copyright holder. To view a copy of this license, visit http://creativecommons.org/ licenses/by/4.0/.

(C) The Author(s) 2019 